\title{
An Autonomous Electrochemical Test stand for Machine Learning Informed Electrolyte Optimization
}

Jay F. Whitacre, ${ }^{*+*}$ Jared Mitchell,, Adarsh Dave, Sven Burke, Venkatasubramanian Viswanathan*s

Department of Materials Science and Engineering, Carnegie Mellon University, 5000 Forbes Avenue, Pittsburgh, Pennsylvania 15213, USA.

${ }^{i}$ Department of Engineering and Public Policy, Carnegie Mellon University, 5000 Forbes Avenue, Pittsburgh, Pennsylvania 15213, USA.

Department of Mechanical Engineering, Carnegie Mellon University, 5000 Forbes Avenue, Pittsburgh, Pennsylvania 15213, USA.

- The Wilton E Scott Institute for Energy Innovation, Carnegie Mellon University, 5000 Forbes Avenue, Pittsburgh, Pennsylvania 15213, USA.

\begin{abstract}
A fully automated, computer-controlled test stand capable of rapidly creating and electrochemically characterizing any arbitrary liquid electrolyte solution is described. Hundreds of different electrolytes were studied, and the results were used to verify the precision and accuracy of the system. To test the functionality of the approach, several 2dimensional co-solvated electrolyte solutions containing blends of aqueous sulfates and nitrates were rapidly created and examined automatically. The test stand took less than a day to conduct these searches, while conventional manual methods would have taken much longer. The demonstrated standard error of the test-stand was $0.5 \mathrm{mS} / \mathrm{cm}$ on conductivity and $0.02 \mathrm{~V}$ for voltage stability window measurements, and several of the combinations studied revealing surprisingly high voltage stability and conductivity values. The demonstrated success of the test-stand in a 2-dimensional search spaces shows the promise of conducting high speed co-optimization studies of liquid electrolytes in particular when used in concert with a machine learning-based real time/in-loop data assessment computational package.
\end{abstract}




\section{Introduction}

There have been multiple reports of experimental methods for the rapid assessment of materials for energy applications, with an increase in high-throughput combinatorial techniques in the 1990's.[1] Specifically, materials for catalysis, photocatalysis, solid state photonic conversion, and energy storage have all been explored, at times with success.[2] Commonly, these studies have focused on solid phase functional materials, with far fewer efforts being devoted to the study of liquid phase materials such as electrolytes for batteries. At the same time, many of the electrolyte systems used, especially for lithium-ion batteries, have multiple co-solvents and additives (along with at least one salt species).[3, 4] With this level of complexity, comprehensively assessing the full range of combinations of constituents and identifying optimal combinations, even with high throughput/combinatorial techniques, is not practical. This is especially true as the number of electrolyte constituents grows, and the problem becomes a nearly untenable manydimensional co-optimization exercise.

Concurrently, the emergence of data-intensive methodologies such as predictive machine learning and other related computational techniques that leverage heretofore unexplored computational power, provide a new opportunity to use data sets that are one or more orders of magnitude larger than have been contemplated by prior generations of researchers. The ongoing work around the materials genome project/movement has also sped up the applications of computationally intensive techniques to the functional materials domain.[58] Recently there has been growth in the field of integrating a high throughputs data acquisition test stand to a data-intensive computational node such that subsequent experimental iterations are based on the data collected and are controlled autonomously. These approaches have found success in the nano/biological where the rapid screening and selection of novel pharmaceuticals or functional molecules has been demonstrated by several groups[9, 10]. To our knowledge, however, there has never been a test stand disclosed that is capable of mixing a completely arbitrary electrolyte solution and assessing various attributes (both physical and electrochemcical) of that liquid in a real time, semicontinuous fashion. 
Such a test stand needs to be able to create an electrolyte solution that is arbitrary in composition, rapidly test multiple characteristics, and then publish the collected data to a server for integration. As such, we have designed and assembled an electrolyte metering/mixing/assessment test stand capable of making and evaluating any liquid electrolyte with up to $n$ distinct constituents, where $n$ is 10 in this case but is, in principle, unlimited. Our first demonstration focuses on the measurement of three key parameters; $\mathrm{pH}$, conductivity, and electrochemically functional voltage stability window, though the addition of many other measurement such as viscosity, electrolyte/electrolyte charge transfer capability, and corrosion currents (to name a few) may be implemented (and will be explored in future work). To demonstrate function and prove out this system, we have chosen to focus on aqueous solvated systems containing salts with differing cations and anions; $\mathrm{Na}$ and $\mathrm{Li}$ cations and $\mathrm{NO}_{3} / \mathrm{SO}_{4}$. We do these because there is virtually no data concerning the range of possible behaviors of mixed cation/anion aqueous solution chemistries (though there is interest in this approach); only several studies with a few data points having been collected to date.[11-14] We also note that the system is completely compatible with organic solvent solutions and we intend to apply this to the study of lithium ion battery electrolytes in the near term.

\section{Experimental}

Figure 1 contains both a simple control flow diagram and pictorial representation of the system. The intended use mode of this system is to have a machine learning software package that incorporates both data collected on the system and information from computational models to inform subsequent experimental steps with the intention of discovering optimal performance parameters based on user-supplied objective functions. This paper is focused on describing the experimental stand that is able to autonomously gather the data and report it back to a shared database, while future publications from our group will describe the integration of this experimental set up with the machine learning platform. We break our system down into two parts: the hardware and software platforms. 


\section{Hardware Platform:}

As shown in the the simple experimental flow diagram in figure 2(a), up to 10 liquid precursors (which are either solvated salt solutions or pure solvents) are contained in individual vessels that feed into a 10-port rotary stream selector valve made by VICI Valco. The valve was directly controlled VICI's universal (USB) power supply/controller. Plumbing was constructed of either $1 / 16$ " or $1 / 8$ " OD ETFE tubing terminating with $1 / 4-28$ UNF flat-bottom flangeless fittings. The output/common port of the valve connected to a high-precision positive-displacement low-volume pump made by Fluid Metering, Inc (FMI). The pump's wetted components were ETFE and alumina and utilized a valveless reciprocating piston design. These pumps allow for precise dosing and flow-rate metering in both forward and reverse directions consistently over millions of cycles. They were controlled via a proprietary Labview module that varied the inputs of pump speed, acceleration, direction, and strokes for each ingredient and step. Flow out of this pump entered a solenoid-controlled 3-way valve made by Cole-Parmer with all PTFE wetted components. Valve switching was mediated by an Agilent 34970a Data Acquisition/Switch Unit and controlled through an open source driver in LabView.

The output of this valve went either (a) straight to the waste bottle (when being flushed/cleaned) or, (b) into the mix vessel. The waste bottle was a 2L HDPE GL45 bottle and the mix vessel was a $10 \mathrm{~mL}$ polished $316 \mathrm{~L} \mathrm{SS}$ walled cylinder affixed to a Thermolyne Maxi-Mix vortex mixer. The outlet port of this mixing vessel taped into the bottom-most point of the internal chamber's conical base ensuring complete extraction of mixed electrolytes and connected to second FMI metering pump. Mixed contents were then transferred into a series of flow-thru devices which measured and recorded ionic conductivity, $\mathrm{pH}$, and voltage stability window.

A Consort C3410 2-channel multi-parameter analyzer performed $\mathrm{pH}$ and conductivity measurements via a Thermo Fisher Orion 9863BN micro pH probe and a Topac K=1.0 4-pole glass/Pt conductivity probe, respectively. A custom PTFE flow through housing was made for both probes. Additionally, the entire conductivity probe fixture was mounted on a buoyancy assistance mechanism that vertically inverted to ensure the chamber 
was filled to eliminate air pockets and also ensure complete drainage. The final components in the system were a flow-through three-electrode cell connected to a Palmsens 4 potentiostat. The three-electrode cell contained two Pt $0.5 \mathrm{~mm}$ wire electrodes (WE/CE) and an $\mathrm{Ag} / \mathrm{AgCl}$ (sat'd $\mathrm{KCl}$ ) reference. Once again, a Labview VI architecture controlled test initiation and data collection using the Palmsens.

To quickly assess the potentials at which the electrolytes undergo electrolysis under anodic and cathodic bias conditions, the electrolyte fluid is subjected to a stair-case potentiometry experiment in the electrochemical test cell; the Palmsens imposed four fixed currents on the working electrode, first cathodic then anodic, for a total of eight current steps. The Palmsens measures the voltage response at the electrode in one-second increments. The fixed current densities are $111,22.2,5.55$, and $1 \mathrm{~mA} / \mathrm{cm}^{\wedge} 2$. The first step is held longer in both the cathodic and anodic steps to promote formation of bilayer and other interface structures. The test runs in a total of 60 seconds, resulting in a fast way to experimentally screen for the electrochemical voltage window of a particular electrolyte formulation. We note that different techniques will yield slightly different absolute values, however we suggest that our approach provides for accurate comparative assessment.

In order to describe the current-voltage response curve with a single value (e.g. for use in machine-learning driven optimization), we calculate tangent lines of this curve joining current steps 2 and $3\left(22.2\right.$ and $\left.5.55 \mathrm{~mA} / \mathrm{cm}^{\wedge} 2\right)$ in both the cathodic and anodic regions. These tangent lines are extrapolated to the zero-current line (i.e. $x$-axis intercept on current-voltage response curve), to generate an estimate for cathode and anode voltage stability. The approach is similar to that reported elsewhere, though in this case it is fully automated and values are obtained in a matter of minutes.[11]

Software platform: The software control architecture of the test-stand is described schematically in Figure 2(b). The experimental hardware is orchestrated by Labview that is run on a dedicated control CPU. This computer also hosts a web server and HTTP endpoints to command the experiment through Labview. All experimental inputs and outputs are communicated over HTTP in a JSON format. The web server logs all input and output data, as well as all relevant meta-data, including ambient temperature, the identity of the 
experimenter, chemical inventory and batch identifiers, potentiometry parameters, and more.

A Python-based application program interface (API) was developed to interface with the web-server, managing all inputs and outputs in a simple manner. Dragonfly, our Bayesian machine learning optimization toolkit used to optimize multicomponent electrolytes, also interfaces with this same Python API to command the test-stand. The implementation of the Dragonfly toolkit is not disclosed in this work, and will be reported in a future publication.

\section{Calibration, Accuracy, and Baseline testing}

Comprehensive control studies were completed to assess the accuracy and precision of teststand measurements. Potential sources of errors included measurement noise (for both the conductivity meter and the electrochemical cell), pumping/volume/metering related errors (due to relative effects of priming and viscosity), and contamination errors (due to potential electrolyte residue left in the closed volumes of the system's plumbing).

Contamination studies: The impact of cross-contamination between contrasting solutions was assessed with a study alternating high and low concentration $\mathrm{KCl}$ solutions, in sets of 8 experimental data points. In this case, conductivity measurements were made while alternating the concentration between 1.0 and $0.1 \mathrm{M}$, doing 4 separate metering and measurements for each of the concentrations.

Conductivity accuracy testing: To assess how repeatable the system when introducing and assessing the conductivities of solutions with contrasting compositions, two concentrations of $\mathrm{KCl}$ conductivity standards $(0.1 \mathrm{M}$ and $1.0 \mathrm{M})$ as well as $\mathrm{K}_{2} \mathrm{SO}_{4} \operatorname{standards}(.1 \mathrm{M}$ and 0.5 M) were used. 75 repeats of the following sequence of concentrations were conducted: 1.0 $\mathrm{M}$ stock solution, $0.1 \mathrm{M}$ stock solution, and finally machine dilution of $0.5 \mathrm{M}$ stock solution down to $0.1 \mathrm{M}$ solution, with each run being done twice with the only second being the intended recorded value (see contamination study). 
Volumetric measurement and pumping errors: The accuracy in delivering repeatable and known amounts of liqud was assessed by repeatedly measuring the conductivity of $0.1 \mathrm{M}$ $\mathrm{KCl}$ made in two different ways: (a) the as-made $0.1 \mathrm{M}$ lab-standard and (b) an autonomously diluted solution starting with a $1.0 \mathrm{M}$ and creating a $0.1 \mathrm{M}$ by mixing with de-ionized water in the test-stand.

Demonstration study:

To demonstrate the range and versatility of the system, we selected several proof-of-concept aqueous-solvated anion and cation pairs. Specifically, electrolyte solutions of mixed lithium and sodium nitrate and sulfate were automatically created that had a wide range of anion and cation ratios. Conductivity and voltage stability values were then extracted using the protocols described above. The full 2-dimensional experimental matrices were measured by taking $1 \mathrm{ml}$ increments of each stock solution and mixing them at the corresponding steps. The test matrix also included dilutions of stock solution in $1 \mathrm{ml}$ increments to set the bounds of the grids. Each individual point measurement took approximately 7 minutes and so the hundreds of distinct experiments represented here were completed in a matter of days.

In doing this set of experiments, we have elected to represent the composition of the electrolyte in terms of molar fraction. Defining molar fraction of a specific solute in a binary solution was done through recording the volume of each stock solution used for a point experiment, the molar mass of each solute, and the molality of each stock solution. These values were used along with equation (1) to determine the mass fraction of a solute in stock solutions. These mass fractions were used along with equation (2) to determine molar fraction of solute in each stock solution.

$$
F_{\text {mass }}=1-\left(\frac{1}{\left(\text { Molal }^{*} \mu+1\right)}\right)
$$




$$
F_{\mu \text { Salt }}=\frac{F_{\text {mass }, \text { Salt }}}{\mu_{\text {Salt }}}
$$

In these equations, Molal is the molal content of a solvated species while $\mu$ is the molecular weight of the salt in question. The volume ratios of stock solutions in each binary solution was then used in conjunction with the molar fractions of those stock solutions to determine specific solute molar fractions for binary solutions.

Table 1 contains a comprehensive listing of the experiments performed in the course of collecting the data published in this work. 
Table1: Listing of experiments performed to both test system integrity and perform simple surveys of mixed anion/cation solutions of varying composition.

\begin{tabular}{|c|c|c|c|c|c|}
\hline$\#$ & Precursor Fluids & Purpose & Description & Values recorded & \# of tests \\
\hline \multirow[b]{2}{*}{1} & $1 \mathrm{M} \mathrm{KCl}$ & \multirow{2}{*}{$\begin{array}{l}\text { To asses the possibility of } \\
\text { contamination between tests } \\
\text { performed in sequence. }\end{array}$} & \multirow{2}{*}{$\begin{array}{c}\text { Alternating between } 4 \\
\text { runs of } 1.0 \mathrm{M} \mathrm{KCl} \text { and } \\
0.1 \mathrm{M} \mathrm{KCl} \text { standards }\end{array}$} & \multirow{2}{*}{$\begin{array}{c}\begin{array}{c}\text { Conductivity and degree } \\
\text { of contamination between } \\
\text { runs }\end{array} \\
\end{array}$} & \multirow[b]{2}{*}{800} \\
\hline & $0.1 \mathrm{M} \mathrm{KCl}$ & & & & \\
\hline \multirow{3}{*}{2} & $1 \mathrm{M} \mathrm{KCl}$ & \multirow{3}{*}{$\begin{array}{l}\text { To verify the accuracy and } \\
\text { repeatability of the conductivity } \\
\text { measurements, as well as ascertain } \\
\text { variance in conductivity of solutions } \\
\text { resulting from mixing solutions. }\end{array}$} & \multirow{3}{*}{\begin{tabular}{|c|} 
Alternating between \\
stock solutions of $1.0 \mathrm{M}$, \\
$0.1 \mathrm{M}$ and mixed to 0.1 \\
$\mathrm{M} \mathrm{KCl}$ solutions
\end{tabular}} & \multirow{3}{*}{$\begin{array}{l}\text { Voltage Stability data, } \\
\text { mixed solution } \\
\text { concentrations }\end{array}$} & \multirow{3}{*}{70} \\
\hline & $0.1 \mathrm{M} \mathrm{KCl}$ & & & & \\
\hline & DI $\mathrm{H}_{2} \mathrm{O}$ & & & & \\
\hline \multirow{3}{*}{3} & $0.5 \mathrm{M} \mathrm{K}_{2} \mathrm{SO}_{4}$ & \multirow{3}{*}{\begin{tabular}{|c|} 
To assess the dependence of the \\
voltage stability window \\
measurement when contrasting \\
solution concentrations are \\
introduced in sequence
\end{tabular}} & \multirow{3}{*}{$\begin{array}{c}\text { Alternating between } \\
\text { stock solutions of } 0.5 \mathrm{M} \text {, } \\
0.1 \mathrm{M} \text { and mixed to } 0.1 \\
\mathrm{M} \mathrm{K}_{2} \mathrm{SO}_{4} \text { solutions, } \\
\text { collect } \mathrm{V} \text { stability data }\end{array}$} & \multirow{3}{*}{$\begin{array}{l}\text { Voltage Stability data, } \\
\text { mixed solution } \\
\text { concentrations }\end{array}$} & \multirow{3}{*}{75} \\
\hline & $0.1 \mathrm{MK}_{2} \mathrm{SO}_{4}$ & & & & \\
\hline & $\mathrm{DI} \mathrm{H}_{2} \mathrm{O}$ & & & & \\
\hline \multirow{3}{*}{4} & 10.0 $\mathrm{M} \mathrm{LiNO}_{3}$ & \multirow{3}{*}{$\begin{array}{l}\text { Binary search for optimal binary } \\
\text { electrolyte solutions in terms of } \\
\text { conductivity and voltage stability } \\
\text { window. }\end{array}$} & \multirow{3}{*}{\begin{tabular}{|c|} 
Binary mixtures of stock \\
solutions and DI water \\
within $7 \mathrm{~mL}$ volumes, \\
changes in volumes \\
across this grid were \\
done in $1 \mathrm{~mL}$ steps. \\
\end{tabular}} & \multirow{3}{*}{\begin{tabular}{|} 
Voltage Stability data, \\
solution conductivity, $\mathrm{pH}$, \\
mixed solution \\
concentrations
\end{tabular}} & \multirow{3}{*}{70} \\
\hline & $7.0 \mathrm{M} \mathrm{NaNO}_{3}$ & & & & \\
\hline & $\mathrm{DI} \mathrm{H} \mathrm{H}_{2} \mathrm{O}$ & & & & \\
\hline \multirow{3}{*}{5} & 10.0 $\mathrm{M} \mathrm{LiNO}_{3}$ & \multirow{3}{*}{$\begin{array}{l}\text { Binary search for optimal binary } \\
\text { electrolyte solutions in terms of } \\
\text { conductivity and voltage stability } \\
\text { window. }\end{array}$} & \multirow{3}{*}{\begin{tabular}{|} 
Binary mixtures of stock \\
solutions and DI water \\
within $7 \mathrm{~mL}$ volumes, \\
changes in volumes \\
across this grid were \\
done in $1 \mathrm{~mL}$ steps.
\end{tabular}} & \multirow{3}{*}{$\begin{array}{l}\text { Voltage Stability data, } \\
\text { solution conductivity, } \mathrm{pH} \text {, } \\
\text { mixed solution } \\
\text { concentrations }\end{array}$} & \multirow{3}{*}{70} \\
\hline & $3.0 \mathrm{M} \mathrm{Li}_{2} \mathrm{SO}_{4}$ & & & & \\
\hline & DI $\mathrm{H}_{2} \mathrm{O}$ & & & & \\
\hline \multirow{3}{*}{6} & $10.0 \mathrm{~m} \mathrm{LiNO}_{3}$ & \multirow{3}{*}{$\begin{array}{l}\text { To act as a binary search for optimal } \\
\text { binary electrolyte solutions. Binary } \\
\text { search for optimal binary electrolyte } \\
\text { solutions in terms of conductivity } \\
\text { and voltage stability window. }\end{array}$} & \multirow{3}{*}{$\begin{array}{l}\text { Binary mixtures of stock } \\
\text { solutions and DI water } \\
\text { within } 7 \mathrm{~mL} \text { volumes, } \\
\text { changes in volumes } \\
\text { across this grid were } \\
\text { done in } 1 \mathrm{~mL} \text { steps. }\end{array}$} & \multirow{3}{*}{$\begin{array}{l}\text { Voltage Stability data, } \\
\text { solution conductivity, } \mathrm{pH} \text {, } \\
\text { mixed solution } \\
\text { concentrations }\end{array}$} & \multirow{3}{*}{70} \\
\hline & $1.5 \mathrm{~m} \mathrm{Na}_{2} \mathrm{SO}_{4}$ & & & & \\
\hline & DI $\mathrm{H}_{2} \mathrm{O}$ & & & & \\
\hline
\end{tabular}

\section{Results and Discussion:}

\section{Precision and Accuracy Assessments}

Figure 3 shows the result of experiment \#1 listed in table 1. The data show that as the system switches between solutions with significantly different electrolyte concentrations (conductivity values), 1 and $0.1 \mathrm{M} \mathrm{KCl}$ solution in this case, it takes 3 measurements to converge on a steady state value. The first of four measurement made after changing electrolyte type was clearly influenced by residual content in the system from the prior 4 measurements, however as subsequent electrolyte is automatically introduced to the test vessel, the measurement value converges to the anticipated steady state value. Based on these results, we assess that a reported data-point is a set of three experimental runs: the first with de-ionized water, the second with the desired fluid blend, and the third being an 
identical repeat of the second; this third run is recorded as the measured value, though data from all experiments are logged and used to assess system behavior retrospectively.

The data contained in figure 4 represents experiment $\# 2$ as listed in table 1 and shows data from repeated conductivity measurements on electrolytes that were either from a pre-mixed $0.1 \mathrm{M}$ solution or from a $0.1 \mathrm{M}$ solution mixed by the system starting with $1 \mathrm{M}$ and adding deionized water. The lab standard conductivity had a very low variability of $0.2 \mathrm{mS} / \mathrm{cm}$ (showing the repeatability of the conductivity measurement), while the system-mixed solution had a variability of $0.4 \mathrm{mS} / \mathrm{c}$. This variation was caused by a combination of the measurement variability shown using the pre-mixed solution and small differences in solution metering that the system introduced. Accounting for the largest ranges of error/variability encountered, we are henceforth able to report a standard error in conductivity measurement as $0.5 \mathrm{mS} / \mathrm{cm}$, which is very reasonable given that most measurements made were in the 10's or 100 's of $\mathrm{mS} / \mathrm{cm}$.

The data in Figure 5 is the outcome from experiment \#3 and is similar to that of experiment $\# 2$ in illustrates volumetric metering error as well as measurement precision, though in this case is for the potentiostat-based voltage stability window measurement on $\mathrm{K}_{2} \mathrm{SO}_{4}$ solution of $0.1 \mathrm{M}$ produced either as a lab standard or automatically by the test stand. The values were bounded by $20 \mathrm{mV}$ on either side. Measurement accuracy is assessed by comparing the test-stand's IV curve to literature results, shown in SI figures. As such we report standard error on voltage window measurements as $0.02 \mathrm{~V}$.

\section{Demonstration study}

The conductivity and voltage window properties of 3 binary salt surveys (experiments \#4-6) are shown in figure 6. To provide a comparative anchor, $\mathrm{LiNO}_{3}$ was present in all three binary surveys. The data show that $\mathrm{NO}_{3}$ containing solutions, especially those with relatively high $\mathrm{LiNO}_{3}$ content, demonstrated superior voltage stabilities when compared to all other dilute solutions (fig. 6D). $\mathrm{NO}_{3}$ containing solutions also demonstrated 
superior conductivities, additionally the $\mathrm{NaNO}_{3}$ solution proved more conductive than $\mathrm{LiNO}_{3}$ solutions (fig. 6C).

The surveys seen in figure 6 also show the conductivity and voltage stability of single salt solutions as a function of concentration. As expected, each solute demonstrated enhanced conductivity and voltage stability at higher concentrations. The $\mathrm{NO}_{3}$ ioncontaining solutions had the highest voltage windows and conductivities. $\mathrm{NaNO}_{3}$ solutions demonstrated the highest conductivities, as well as the highest solute molar fractions. To compare solutions of $\mathrm{NaNO}_{3}$ and $\mathrm{LiNO}_{3}$, the molar fraction of each solute was compared at a point with a similar conductivity. $\mathrm{A} \mathrm{NaNO}_{3}$ solution with a conductivity was $148.1 \mathrm{mS} / \mathrm{cm}$ represented 6.04 molar\%, while a $\mathrm{LiNO}_{3}$ solution with a conductivity of $148.0 \mathrm{mS} / \mathrm{cm}$ was 7.84 molar\%, suggesting that $\mathrm{NaNO}_{3}$ solutions are more conductive than $\mathrm{LiNO}_{3}$ solutions per mole of solute. Regardless of the molar fraction the $\mathrm{NO}_{3}$ ion containing solutions were more conductive and had larger voltage windows than $\mathrm{SO}_{4}{ }^{2}$ containing solutions.

Figure 6A exhibited conductivity values for mixed $\mathrm{LiNO}_{3}$ and $\mathrm{Li}_{2} \mathrm{SO}_{4}$ solutions that had marked improvements at dilute concentrations of both solutes, while at higher concentrations the mixed solutions exhibited conductivities more similar to single solute $\mathrm{LiNO}_{3}$ solutions, suggesting that $\mathrm{NO}_{3}$ is the dominant anion. While the $\mathrm{NO}_{3}$ ion is more prevalent in higher concentration mixed solutions, dilute mixed solutions also exhibited conductivities more similar to single solute $\mathrm{LiNO}_{3}$ solutions.

The binary $\mathrm{LiNO}_{3}$ and $\mathrm{NaNO}_{3}$ solutions shown in figures $6 \mathrm{C}$ and $6 \mathrm{D}$ exhibited increased conductivity with increased solute molar fractions; at higher concentrations of solute, the conductivity was dominated by the more soluble $\mathrm{NaNO}_{3}$. At lower concentrations, solution conductivities over $140 \mathrm{mS} / \mathrm{cm}$ were reached quickly; however, conductivities of pure $\mathrm{LiNO}_{3}$ solutions were higher than those of the mixed solutions for comparable solute molar fractions. The binary $\mathrm{LiNO}_{3}$ and $\mathrm{NaNO}_{3}$ solutions exhibited increases in conductivity and voltage window that appear to emerge from the mixing of the solute species in solution. This is most noticeable at the $\left(0.016 \mathrm{LiNO}_{3}, 0.106 \mathrm{NaNO}_{3}, 2.83\right.$ $\mathrm{V}, 172.7 \mathrm{mS} / \mathrm{cm}$ ) peak; only in mass fractions above 0.128 did single solute $\mathrm{NaNO}_{3}$ solutions reach above $2.8 \mathrm{~V}$ windows, and the highest voltage window reported for the single solute $\mathrm{LiNO}_{3}$ solutions was $2.77 \mathrm{~V}$. Other such increases in voltage window are present at the points $\left(0.0500 \mathrm{LiNO}_{3}, 0.085 \mathrm{NaNO}_{3}, 2.81 \mathrm{~V}, 163.8 \mathrm{mS} / \mathrm{cm}\right)$, and $\left(0.066 \mathrm{LiNO}_{3}, 0.063 \mathrm{NaNO}_{3}\right.$, 
$2.84 \mathrm{~V}, 160 \mathrm{mS} / \mathrm{cm})$. At total solute molar fractions of around $0.122,0.135,0.129$ these points have higher voltage windows than the corresponding pure $\mathrm{NaNO}_{3}$ stock solution, suggesting that ion concentration is not only variable of significance. While the first of these peaks mentioned likely falls within the margin of error, the other peaks are highly encouraging. These points also illustrate conductivity decreasing with the decreasing mass fractions of $\mathrm{NaNO}_{3}$. There appears to be no trend for voltage window among these three peaks.

Binary mixture solutions in figure $6 \mathrm{C}$ demonstrated that the performance of the $\mathrm{LiNO}_{3}$ tended to be dominate over the $\mathrm{Na}_{2} \mathrm{SO}_{4}$. Conductivity values tended to be higher in the binary mixed region than for either single solute solution; however, the voltage windows were on par with those exhibited by single solute $\mathrm{LiNO}_{3}$ solutions

\section{Summary/Conclusion:}

A novel design was used to build an experimental test-stand that was then controlled by a custom software package capable of mixing and electrochemically characterizing electrolyte solutions autonomously. Rapid testing of hundreds of different aqueous solutions of sodium and/or lithium nitrate and/or sulfate using the stand was used to carry out control studies. The demonstrated standard error of the test-stand was $0.5 \mathrm{mS} / \mathrm{cm}$ on conductivity and $0.02 \mathrm{~V}$ on voltage stability window measurements. The test-stand was then used to query several 2-dimensional electrolyte search spaces discovering novel binary electrolyte solution blends such as aqueous LiNO3 and NaNO3 blends with voltage stabilities exceeding $2.8 \mathrm{~V}$. The test-stand took less than a day conduct these searches while conventional manual methods would likely have taken much more time. The demonstrated success of the test-stand in these 2-dimensional search spaces merits its wider use in searching the complex, high-dimensional and expensive to search design space of electrochemistry, in particular when used in concert with a machine learning based real time/inloop data assessment computational package. 
Acknowledgements: This work was funded by the Toyota Research Institute under the AMDD program, with some additional support from the Scott Institute for Energy Innovation at Carnegie Mellon University 


\section{References}

1. Xiang, X.-D., et al., A combinatorial approach to materials discovery. Science, 1995. 268(5218): p. 1738-1740.

2. Rajan, K., Combinatorial materials sciences: Experimental strategies for accelerated knowledge discovery. Annu. Rev. Mater. Res., 2008. 38: p. 299-322.

3. Aurbach, D., et al., Design of electrolyte solutions for Li and Li-ion batteries: a review. Electrochimica Acta, 2004. 50(2-3): p. 247-254.

4. Zhang, S.S., A review on electrolyte additives for lithium-ion batteries. Journal of Power Sources, 2006. 162(2): p. 1379-1394.

5. Fujimura, K., et al., Accelerated Materials Design of Lithium Superionic Conductors Based on First-Principles Calculations and Machine Learning Algorithms. Advanced Energy Materials, 2013.3(8): p. 980-985.

6. Butler, K.T., et al., Machine learning for molecular and materials science. Nature, 2018. 559(7715): p. 547.

7. Meredig, B., et al., Combinatorial screening for new materials in unconstrained composition space with machine learning. Physical Review B, 2014. 89(9): p. 094104.

8. Botu, V. and R. Ramprasad, Adaptive machine learning framework to accelerate ab initio molecular dynamics. International Journal of Quantum Chemistry, 2015. 115(16): p. 10741083.

9. King, R.D., et al., Functional genomic hypothesis generation and experimentation by a robot scientist. Nature, 2004. 427(6971): p. 247.

10. Nikolaev, P., et al., Discovery of wall-selective carbon nanotube growth conditions via automated experimentation. ACS nano, 2014. 8(10): p. 10214-10222.

11. Wessells, C., et al., Investigations of the electrochemical stability of aqueous electrolytes for lithium battery applications. Electrochemical and Solid-State Letters, 2010. 13(5): p. A59-A61 .

12. Wang, Y., J. Yi, and Y. Xia, Recent Progress in Aqueous Lithium-Ion Batteries. Advanced Energy Materials, 2012. 2(7): p. 830-840.

13. Suo, L., et al., Advanced high-voltage aqueous lithium-ion battery enabled by "water-inbisalt" electrolyte. Angewandte Chemie International Edition, 2016. 55(25): p. 71367141. 
14. Suo, L., et al., “Water-in-salt” electrolyte enables high-voltage aqueous lithium-ion chemistries. Science, 2015. 350(6263): p. 938-943. 


\section{Figure Captions}

Figure 1: Control flow diagram of full test apparatus. The test-stand is orchestrated by a Labview web server on a nearby Control CPU, commanded over HTTP requests by a Python API. The machine-learning software plugs into the Python API to order experiments. Electrolyte models are fed into the machine-learning software as statistical priors. All data and meta-data are saved on a cloud database.

Figure 2: (a) One-line flow diagram of the autonomous liquid electrolyte mixing/test system. (b) Pictorial representation of all system components. 1: Vented glass bottle w/ pickup tube (x10), 2: 10 position rotary valve 3: Calibrated positive displacement pump (Dosing) 4: 3-way valve, 5: Waste container, 6: Vortex mixer, 7: Calibrated positive displacement pump (Transfer), 8: 4-pole ionic conductivity probe w/ flow-thru cell, 9: Buoyancy assistance inversion mechanism, 10: Pt-Pt-Ag/AgCl flow-thru e-chem cell, 11: Waste container

\section{Figure 3:}

Mixed solution conductivity data for two different concentrations of $\mathrm{KCl}$ solvated in water. The experiments conducted consisted of a repeating loop of 8 conductivity measurements, four on the $1 \mathrm{M}$ concentration solution followed by four on the $0.1 \mathrm{M}$ concentration solution. The first measurement after changing concentration was an expected outlier as some of the solution from the previous run was left in the system. The Blue, dotted lines indicate standard conductivity values for each formulation at $20 \mathrm{C}$. The test-stand converges to within one-standard error $(0.5 \mathrm{mS} / \mathrm{cm})$ of true value at the second run.

Figure $4: \mathrm{KCl}$ control study comparing a lab-made $0.1 \mathrm{M}$ solution with a $1.0 \mathrm{M}$ solution diluted to $0.1 \mathrm{M}$ by the test-stand. Black, dotted lines indicate confidence bounds of onestandard-error above and below the mean. The test-stand accurately mixes and measures solution conductivity, and with confidence bounds of $0.2 \mathrm{mS} / \mathrm{cm}$. 
Figure 5: Voltage stability window measurement study results using $0.1 \mathrm{M} \mathrm{K}_{2} \mathrm{SO}_{4}$ solutions that were both pre-prepared and produced automatically by the test-stand. Black, dotted lines indicate confidence bounds of one-standard-error above and below the mean. The test-stand accurately mixes and measures the stability window, and with confidence bounds of $20 \mathrm{mV}$.

Figure 6. Results from two-dimensional scattered data of conductivity and voltage window vs. electrolyte composition (salt mass fraction) taken by the test-stand. Conductivity is represented by the color axis for figures (a), (c), and (e3) on the left. Voltage is represented color axis for figures (b), (d), and (f) on the right. The black dots on all figures represent the data points as gathered by the test stand, the colored contour map represents interpolation of the data. X and Y-axes represent the molar fractions of solutes. (a) - conductivity map of the mixed cation case where binary mixtures of aqueous $\mathrm{Li}_{2} \mathrm{SO}_{4}$ and $\mathrm{LiNO}_{3}$ solutions are used; (b) - voltage stability map of the mixed cation case where binary mixtures of aqueous $\mathrm{Li}_{2} \mathrm{SO}_{4}$ and $\mathrm{LiNO}_{3}$ solutions are used; (b) - conductivity map of mixed anion case where binary mixtures of aqueous $\mathrm{LiNO}_{3}$ and $\mathrm{NaNO}_{3}$ solutions are used; (d) - voltage stability map of the mixed mixed anion case where binary mixtures of aqueous $\mathrm{LiNO}_{3}$ and $\mathrm{NaNO}_{3}$ solutions are used; (e) - conductivity map of mixed cation and anion case where aqueous $\mathrm{LiNO}_{3}$ and $\mathrm{Na}_{2} \mathrm{SO}_{4}$ solutions are used; (f)- voltage stability map of mixed cation and anion case where aqueous $\mathrm{LiNO}_{3}$ and $\mathrm{Na}_{2} \mathrm{SO}_{4}$ solutions are used. The error for voltage and conductivity are constant across all test and are $\pm 0.02 \mathrm{~V}$ and $\pm 0.5 \mathrm{mS} / \mathrm{cm}$ respectively. See SI figure for three-dimensional representations. 